\title{
Survey on Multi-Hop Communication To Enhance The Efficiency Of Sensor Node in WSN
}

\author{
Piyush Mahajan', Navjeet Saini' ${ }^{2}$, Deepak Kumar ${ }^{3}$ \\ ${ }^{1}$ Sri Sai College Of Engineering and Technology, \\ Badhani Pathankot Punjab, India \\ ${ }^{2}$ Sri Sai College Of Engineering and Technology \\ Badhani Pathankot Punjab, India \\ ${ }^{3}$ Sri Sai College Of Engineering and Technology \\ Badhani Pathankot Punjab, India
}

\begin{abstract}
:
In WSN routing is the primary task for data communication between $\mathrm{CHs}$ to $\mathrm{BS}$. The routing algorithm used should be energy efficient so that it can surmount related power constraints. As LEACH is energy efficient cluster based routing protocol used in Wireless sensor network, there are many version of LEACH one of them is MODLEACH. MODLEACH introducing efficient cluster head replacement scheme and dual transmitting power levels, However the efficiency of the MODLEACH can further be increased by introducing certain changes in the $\mathrm{CH}$ selection process and Multi-Hop communication between $\mathrm{CH}$ and $\mathrm{BS}$. The cluster head selection is based on the probability and the threshold equation, but this may be problem for the next round. There is no concept that, it is based on nth power of energy conservation technique because cluster head selection not consider any energy based parameter like residual energy of sensor node to be formed as $\mathrm{CH}$.
\end{abstract}

Keywords: WSN, Sensors, multi-hop, Clustering.

\section{Introduction}

In WSNs, the only source of life for the nodes is the battery. Communicating with other nodes or sensing activities consumes a lot of energy in processing the data and transmitting the collected data to the sink. In many cases (e.g. surveillance applications), it is undesirable to replace the batteries that are depleted or drained of energy [4]. Many researchers are therefore trying to find energy-aware protocols for wireless sensor networks in order to overcome such energy efficiency problems as those stated above.

All the protocols that are designed and implemented in WSNs should provide some real-time support as they are applied in areas where data is sensed, processed and transmitted based on an event that leads to an immediate action. A protocol is said to have real-time support if and only if, it is fast and reliable in its reactions to the changes prevailing in the network. It should provide redundant data to the base station. The base station or sink using the data that is collected among all the sensing nodes in the

network. The delay in transmission of data to the sink from the sensing nodes should be small, which leads to a fast response.

This paper, proposed energy efficient multi-hop data communication approach in the context of clustering, where cluster heads are selected on the basis of remaining energy of sensor nodes and probability parameters. It increases the stability period of the network because cluster head needs more energy than normal nodes.

\section{Literature Review}

D. Mahmood and N. Javaid et al "MODLEACH: A Variant of LEACH for WSNs". This paper gives birth to a new version of LEACH protocol Called MODLEACH protocol. This paper defines the drawbacks of LEACH protocol according to soft and hard threshold implementation for energy utilization. According to author MODLEACH performs better considering metrics of throughput, network life time, and optimized cluster head formation of network. MODLEACH is further improved by using the 
concept of soft and hard threshold. But the paper did not define the cluster head selection depends upon the probability and data transferred unmannered way not define any route.

Muhammad Nasir Khan and Mohsin Jamil," Performance Improvement in Lifetime and Throughput of LEACH",[2016]. This paper proposes a modified algorithm to improve the performance and life time of sensor nodes. Lifetime and throughput are crucial parameters discussed in the paper. The critical parameters of a WSN protocol Low Energy Adaptive Clustering Hierarchal $(\mathrm{LEACH})$ are improved and the results are compared with original LEACH protocol. In this paper an efficient clustering scheme (En-LEACH) has been proposed which takes into account the residual energy after each round to select new cluster head or not and set the different energy levels for different communications.

Shahina Sheikh, Ms. Hemlata Dakhore, " Data Compression Techniques for Wireless Sensor Network", [2015]. This paper focused on the adaptive Lossless data compression on WSN. This research work reports the simulation \& implementation of efficient data compression algorithms for wireless sensor network. This paper is based on the principle of Adaptive Huffman Code, a novel compression algorithm for WSNs.

Karthick .K et al, "An Energy-Saving Routing Algorithm for Wireless Body Sensor Network using Data Compression Technique", [2015]. In this paper the method Energy consumption leach-c protocol and LZW compression technique is introduced to enhance the lifetime of the sensor node. The sensor node selects their cluster head based on their energy level, the higher energy is represented as the cluster head. In data compression technique only less amount of energy has been consumed during the transmission of the data thus the energy is saved and which increases life time of the sensor node.

Shekhar Vyas1, Pinaki A. Ghosh, " Review and Proposed Work for MODI-LEACH for Improvement of LEACH for Energy Consumption in Wireless Sensor Networks", [2014]. This paper presents the cluster formation protocol using in wireless sensor network. It explains the working of clustering protocol which consists of two phases, first phase of protocol chooses appropriate node for becoming cluster head of particular cluster and In second phase member nodes of cluster send data to cluster head. The aggregation function is used to send all data to base station. This paper proposed protocol MODI-LEACH which deals with energy consumption in wireless sensor network and eliminate redundancy so that redundant data is eliminated.

Chunyao FU et al. "An Energy Balanced Algorithm of LEACH Protocol in WSN." [2013]. This paper focused that due to the drawback of nodes energy, energy efficiency has a significant issue that should be consider while the protocols are designed in WSNs. LEACH Protocol plays a main part as a distinctive representative of hierarchical routing protocols. In response to the irregular energy distribution that has been caused by the uncertainty of cluster heads forming. A novel improved algorithm of LEACH protocol (LEACH-TLCH) has been proposed to balance the energy consumption of the whole network and expand the life of the network.

Yang Jing et al., "An improved routing algorithm based on LEACH for wireless sensor networks." [2013]. The author has been concentrated that LEACH has a good solution for energy efficiency as a representative routing algorithm of hierarchy for WSNs. Although, LEACH has few disadvantages such as complex formation of clustering and direct communication between all cluster heads as well as sink node to transmit high energy consumption. An improved routing algorithm based on LEACH called ILEACH has been proposed. Firstly, the ILEACH employ the residual energy to form clustering which can avoid the low energy node to become a cluster head. Secondly, an energy function has been proposed to balance the energy consumption among cluster heads. Lastly, a data aggregation tree has been constructed to transmit the data from the cluster heads to sink node.

Mr. Santosh. Irappa.Shirol et al, "Advanced-LEACH Protocol of Wireless Sensor network", [2013]. This paper explains the improvement on LEACH protocol based on different parameters such as Remaining Energy, Centrality of the node and Proximity to Base Station. These parameters effects on the selection of cluster heads and the scheme is simulated using Matlab. The Simulation results show the improvement on LEACH protocol and increases the Network life time. It uses the MultiHop communication for data communication between $\mathrm{CH}$ and $\mathrm{BS}$.

Chunyao FU, Zhifang JIANG, Wei WEI and Ang WEI, "An Energy Balanced Algorithm of LEACH 
Protocol in WSN", [2013]. This paper represents a new improved algorithm of LEACH protocols called LEACH-TLCH This Protocol balance the energy consumption of the entire network and extend the network life time and the protocol is simulated in Matlab. The selection of the cluster head is based on energy level of a node and improves the random selection of the nodes.

Zahra Beiranvand et al., "I-LEACH: An efficient routing algorithm to improve performance \& to reduce energy consumption in Wireless Sensor Networks." [2013] has been discussed that a large amount of energy in nodes of a WSN has been consumed due to the inner-network communications. An energy efficient routing algorithm has been proposed which save a major part of inner-network communications energy. The proposed routing algorithm selects sensor nodes among higher residual energy, more neighbors with lower distance from the Base Station (BS) as Cluster Head $(\mathrm{CH})$ nodes. After that it manages sensor nodes properly and constructs clusters to maximize WSN lifetime and minimize average energy dissipation for each sensor node.

Brar and Malhotra [2013] have represented that wireless network has witness an explosion of concern from consumers in latest years for its applications in mobile and personal communications. Wireless networks are an essential component of the current communication infrastructure and energy efficiency has a main design concern due to the limited battery life of mobile terminal. Generally, power conservation techniques are usually used in the hardware design of such systems. As the network interface has an important consumer of power, a lot of research has been devoted to low-power design of the full network protocol stack of wireless networks in an effort to improve energy efficiency. A complete summary of current work addressing energy efficient and low-power design within all layers of the wireless network has been presented and moreover, the guideline for energy conservation have provided. Jia Xu et al., "Improvement of LEACH protocol for WSN." [2012] have presents that sensor nodes forever have a limited power resource in WSNs. The energy consumed by transfer data from the sensor node to its destination raise as a critical problem in design WSN routing protocols. A cluster routing algorithm named E-LEACH to enhance the hierarchical routing protocol $\mathrm{LEACH}$ has been proposed. In the E-LEACH algorithm, the new way of the selection of the cluster heads is random and the round time for the selection is fixed. In the ELEACH algorithm, the residual power of the sensor nodes in order to balance network loads as well as change the round time depends on the optimal cluster size.

Insoo Koo et al., "Performance analysis of ILEACH and LEACH protocols for wireless sensor networks." [2012] have observe the problems of the low energy adaptive clustering hierarchy (LEACH) protocol along with current ideas for enhancement by select the cluster head node. The major problem with LEACH has the random selection of cluster heads. The formed cluster heads are unbalanced and may remain in one part of the network which makes some part of the network inaccessible. The improved LEACH (ILEACH) protocol has been presented in which a cluster head is select based on its ratio among the current energy level and an initial energy level and multiplies by the root square of its number of neighbor nodes.

Yi Liu et al. [2012] have proposed a low energy uneven cluster protocol design. It Aim at the random selection for cluster head of traditional Leach protocol, and the fault of the single hop from the entire cluster heads to the sink node, an improved method for Leach protocol has advanced. Initially, the election model of cluster head has improved and the node residual energy has been considered in the method of threshold and the cluster head election to improve the whole network life cycle. In the multihop route, choose the maximum energy and the nearest node as the next hop and a route transfer data among many clusters has been formed.

M. Aslam et al.,. "A Low Energy Uneven Cluster Protocol Design for Wireless Sensor Network" [2012] have represented that an energy efficient routing protocol is the major concern with WSNs. Some energy efficient hierarchal routing protocols developed from conventional LEACH routing protocol has been presented. It focuses on how these extended routing protocols work in order to increase the lifetime and how quality routing protocol is improved for WSN.

Muhammad Haneef et al., "MG-LEACH: Multi group based LEACH an energy efficient routing algorithm for Wireless Sensor Network" [2012] has been presents that limited energy resource is the major limitation related with WSN. As communication is the main cause of energy 
reduction in the network so designing of energy efficient routing algorithm is the key challenges that have to address for extending life time of network. It has taken deploy redundant nodes into explanation which covers major part of energy reduction in the network. It presents energy efficient routing algorithm based on the structure of LEACH. A lot of redundant data is available in WSN due to usually deployed nodes. The redundancy of deployed nodes can be used as an improvement for increase network lifetime.

Dr. G. T. Raju et al., "Wireless sensor network lifetime optimization" [2011] have presented the WSN for environmental monitoring with optimized lifetime. The node have equipped with multimode sensors for sensing different environmental parameters. An efficient utilization of power has been essential in order to use networks for long duration. Hence, it has been needed to reduce data traffic inside sensor networks with reduce amount of data that need to send to sink. The different strategy to maximize the WSN lifetime includes routing, data aggregation, data accuracy and energy consumption has been studied. The main scheme has to describe a multi metrics protocol that takes into description the remaining energy in sensor nodes with data aggregation and data accuracy.

Yuling $\mathrm{Li}$ et al., "The improvement of LEACH protocol in WSN" [2011] have discussed that WSN has been widely use in many fields such as environmental monitoring, military defense etc. However, the important limitation has energy. Thus, WSN approximately focus on how to save energy. The most traditional algorithm is LEACH. A new improve method called LEACH-N based on LEACH has been proposed. According to improved protocol, the problem shows that how to choose nodes as the cluster head node depends upon the residual energy of nodes in the cluster. This approach definite the rationality during selecting head nodes. Moreover, the network robustness can be improved and the life cycle for the network can be extended.

Ranganathan Vidhyapriya and Ponnuamy Vanathi, "Energy Efficient Data Compression in Wireless Sensor Networks",[2009]. This paper describes two lossless data compression algorithms integrated with the shortest path routing technique to reduce the raw data size and to accomplish optimal trade-off between rate, energy, and accuracy in a sensor network. Paper explains data sharing among sensor nodes with respective to the energy efficiency of sensor nodes. To accomplish this task the nodes are deployed with respect to the energy of a node and compression technique is applied to data sets on a node.

FAN Yiming and YU Jianjun, "The communication protocol for wireless sensor network about LEACH" [2007] has shown that the communication protocol named LEACH which focuses on reducing the power consumption of wireless sensor networks that can have significant impact on the overall energy dissipation of these networks. LEACH uses localized coordination to enable scalability and robustness for dynamic networks, and incorporate data fusion into the routing protocol to reduce the amount of information that must be transmitted to the base station.

Simrandeep kaur, V.Sulochana Verma," Design and Implementation of LZW Data Compression Algorithm". In this paper, LZW data compression algorithm is implemented by finite state machine. This paper gives the idea about the compression technique used in wireless sensor networks. This paper explains how a simple data sets can be compression by using recent lzw compression technique, the data is compression based on the simple compression ratio which can be easily applied on the data packets during transmission.

Jaswant Singh Raghuwanshi, Neelesh Gupta, Neetu Sharma,.In the paper reclustering and multihop data transmission processes are utilized for data reporting to base station by sensor node. The accurate use of energy in WSNs is the main challenge for exploiting the network to the full extent. The main aim of the proposed method is that by evenly distributing the energy all over the sensor nodes and by reducing the total energy dissipation, the lifetime of the network is enhanced, so that the node will remain alive for longer times inside the cluster. A new threshold has been formulated for cluster head selection which is based on the remaining energy of the sensor node and the distance from base station. The result shows that the proposed clustering approach has higher stable region and network life time than TopologyControlled Adaptive Clustering (TCAC) and LowEnergy Adaptive Clustering Hierarchy (LEACH) for WSNs.

\section{Problem Formulation and Proposed Work}

In WSN routing is the primary task for data communication between $\mathrm{CHs}$ to $\mathrm{BS}$. The routing algorithm used should be energy efficient so that it 
can surmount related power constraints. As LEACH is energy efficient cluster based routing protocol used in Wireless sensor network, there are many version of LEACH one of them is MODLEACH. MODLEACH introducing efficient cluster head replacement scheme and dual transmitting power levels, However the efficiency of the MODLEACH can further be increased by introducing certain changes in the $\mathrm{CH}$ selection process and Multi-Hop communication between $\mathrm{CH}$ and $\mathrm{BS}$. The cluster head selection is based on the probability and the threshold equation, but this may be problem for the next round. There is no concept that, it is based on nth power of energy conservation technique because cluster head selection not consider any energy based parameter like residual energy of sensor node to be formed as $\mathrm{CH}$.

Also the data transfer from $\mathrm{CH}$ to Sink or BS does not consider any compressing technique and is in unmannered way.

The proposed work will be based on following objective:-

a) To implement the base paper.

b) To achieve the better Cluster head selection by using residual energy concept with threshold energy equation.

c) To implement multi-hop concept to enhance the efficiency the life time of sensor node.

d) To use the reliable path for better data transfer.

By doing these objectives it will improve the overall WSN life time and energy consumption with sensor node.

\section{References}

[1] FU, Chunyao, Zhifang JIANG, and Wei WEI2and Ang WEI. "An Energy Balanced Algorithm of LEACH Protocol in WSN." International Journal of Computer Science (2013).

[2] Jing, Yang, Li Zetao, and Lin Yi. "An improved routing algorithm based on LEACH for wireless sensor networks." Control and Decision
Conference (CCDC), 2013 25th Chinese.IEEE, 2013.

[3] Beiranvand, Zahra, Ahmad Patooghy, and Mahdi Fazeli. "I-LEACH: An efficient routing algorithm to improve performance \& to reduce energy consumption in Wireless Sensor Networks." Information and Knowledge Technology (IKT), 2013 5th Conference on. IEEE, 2013.

[4] Xu, Jia, Ning Jin, Xizhong Lou, Ting Peng, Qian Zhou, and Yanmin Chen. "Improvement of LEACH protocol for WSN.", In Fuzzy Systems and Knowledge Discovery (FSKD), 2012 9th International Conference on, pp. 2174-2177. IEEE, 2012.

[5] Sipon, Miah Md, and Koo Insoo. "Performance analysis of ILEACH and LEACH protocols for wireless sensor networks." Journal of information and communication convergence engineering 10.4 (2012): 384-389.

[6] Liu, Yi, Shan Zhong, Licai You, Bu Lv, and Lin Du. "A Low Energy Uneven Cluster Protocol Design for Wireless Sensor Network." Int'1 J. of Communications, Network and System Sciences 5 (2012): 86.

[7] Haneef, Muhammad, Zhou Wenxun, and Zhongliang Deng. "MG-LEACH: Multi group based LEACH an energy efficient routing algorithm for Wireless Sensor

Network." Advanced Communication Technology (ICACT), 2012 14th International Conference on. IEEE, 2012.

[8] Aslam, M., Nadeem Javaid, A. Rahim, U. Nazir, Ayesha Bibi, and Z. A. Khan. "Survey of extended LEACH-Based clustering routing protocols for wireless sensor networks."In High Performance Computing and Communication \& 2012 IEEE 9th International Conference on Embedded Software and Systems (HPCCICESS), 2012 IEEE 14th International Conference on, pp. 1232-1238. IEEE, 2012.

[9] Maraiya, Kiran, Kamal Kant, and Nitin Gupta. "Wireless sensor network: a review on data aggregation." International Journal of Scientific \& Engineering Research 2.4 (2011): 1-6.

[10]Raju, G. T., D. K. Ghosh, T. Satish Kumar, S. Kavyashree, and V. Nagaveni. "Wireless sensor network lifetime optimization." (2011): 244248.

[11]Li, Yuling, Luwei Ding, and Feng Liu. "The improvement of LEACH protocol in WSN." Computer Science and Network Technology (ICCSNT), 2011 International Conference on. Vol. 2.IEEE, 2011. Available 
from

http://ieeexplore.ieee.org/document/6233907/

[12]Jung, W. S., Lim, K. W., Ko, Y. B., \& Park, S. J. (2009, February). A hybrid approach for clustering-based data aggregation in wireless sensor networks. In Digital Society, 2009. ICDS'09.Third International Conference on (pp. 112-117). IEEE.

[13]Marta, Mirela, and Mihaela Cardei. "Using sink mobility to increase wireless sensor networks lifetime." World of Wireless, Mobile and Multimedia Networks, 2008. WoWMoM 2008.2008 International Symposium on a. IEEE, 2008.

[14]Basagni, Stefano, Alessio Carosi, Emanuel Melachrinoudis, Chiara Petrioli, and Z. Maria Wang. "Controlled sink mobility for prolonging wireless sensor networks lifetime." Wireless Networks 14, no. 6 (2008).

[15]Yiming, Fan, and Yu Jianjun."The communication protocol for wireless sensor network about LEACH." Computational Intelligence and Security Workshops, 2007.CISW 2007.International Conference on. IEEE, 2007.

[16]Mr. Santosh.Irappa.Shirol, Ashok Kumar. N, Mr. Kalmesh.M.Waderhatti,"AdvancedLEACH Protocol of Wireless Sensor network", IJETT - Volume4 Issue6- June 2013.

[17]Chunyao FU, Zhifang JIANG, Wei WEI and Ang WEI," An Energy Balanced Algorithm of LEACH Protocol in WSN", IJCSI-2013.

[18]Rajashree.V.Biradar (1), V.C .Patil (2), Dr. S. R. Sawant (3) , Dr. R. R. Mudholkar, "CLASSIFICATION AND COMPARISON OF ROUTING PROTOCOLS IN WIRELESS SENSOR NETWORKS", UbiCC Journal.

[19]Ranganathan Vidhyapriya and Ponnusamy Vanathi, "Energy Efficient Data Compression in Wireless Sensor Networks", iajit-2009.

[20]Simrandeep kaur, Student1 ; V.Sulochana Verma," Design and Implementation of LZW Data Compression Algorithm", (IJIST) V

[21]Muhammad Nasir Khan* and Mohsin Jamil," Performance Improvement in Lifetime and Throughput of LEACH", ijst 2016.

[22]Dheemanth H N," LZW Data Compression", AJER-2014.

[23]Md. Sipon Miah and Insoo Koo," Performance Analysis of ILEACH and LEACH Protocols for Wireless Sensor Networks", jicce-2012.

[24]Shekhar Vyas1,Pinaki A. Ghosh2, " Review and Proposed Work for MODI-LEACH for
Improvement of LEACH for Energy Consumption in Wireless Sensor Networks", JAERD-2014.

[25]Shahina Sheikh, Ms. Hemlata Dakhore, " Data Compression Techniques for Wireless Sensor Network", IJCSIT-2015.

[26]Shikha Gupta, Shikha Khanna,"SURVEY OF LEACH AND RELATED PROTOCOLS UNDER WIRELESS SENSOR NETWORKS", IJRREST-2015.

[27] MS. Manisha Yadav, Mrs. Moumita Ghosh," Performance analysis of different types of LEACH protocol depending on various parameters", IRJET-2016.

[28]Karthick .K et al, " An Energy-Saving Routing Algorithm for Wireless Body Sensor Network using Data Compression Technique", ijca-2015.

[29] Satyajeet R. Shinge, "Increase the Lifetime of Wireless Sensor Network using Clustering and Compression", ijca-2015.

[30] S.Mohamed Saleem, "Evaluating Effectiveness of Data Transmission and Compression Technique in Wireless Sensor Networks", ijarcsse-2013.

[31]R. Swathi, “An Energy Efficient Routing Algorithm based on Data Compression in LEACH-C", ijert-2014.

[32]Priyanka1, Navjeet saini2, Sukhbeer Singh3 ," Enhanced MODLEACH Using Effective Energy Utilization Technique for Wireless Sensor Network", International Journal Of Engineering And Computer Science, Volume 5 Issue 09 September 2016 Page No.1816918174. 